\begin{tabular}{ll} 
Abstract 43 Table 1 & Pacing indication \\
\hline Pacing Indication & Number of Patients (Total 21) \\
\hline Sinus Pauses & $7(33 \%)$ \\
Congenital AV Block & $5(24 \%)$ \\
Mobitz II AV Block & $3(14 \%)$ \\
Complete Heart Block & $2(9.5 \%)$ \\
Tachy-Brady Syndrome & $2(9.5 \%)$ \\
Mobitz I AV Block & $1(5 \%)$ \\
AV Node Ablation & $1(5 \%)$ \\
\hline
\end{tabular}

Abstract 43 Table 2 Implantation and follow-up parameters

\begin{tabular}{llll}
\hline & & $\begin{array}{l}\text { Parameters at } \\
\text { Implantation }\end{array}$ & $\begin{array}{l}\text { Parameters at } \\
\text { Follow-Up }\end{array}$ \\
\hline Pacing threshold $(\mathrm{V})$ & Mean & $0.58 \pm 0.31$ & $0.77 \pm 0.7$ \\
& Range & $0.25-1.38$ & $0.38-2.88$ \\
R wave amplitude & Mean & $10.64 \pm 4.31$ & $13.7 \pm 5.8$ \\
$(\mathrm{mV})$ & Range & $4.5-20.0$ & $2.6->20.0$ \\
Impedence $(\Omega)$ & Mean & $813 \pm 175$ & $558 \pm 102$ \\
& Range & $510-1080$ & $430-760$ \\
\hline
\end{tabular}

since first implant (Median duration $15.7 \pm 8.3$ months). There were no cases of device migration or failure. Mean $\mathrm{R}$ wave sensing was $13.7 \pm 5.8 \mathrm{mV}$. 16 of the 18 patients had a threshold $\leq 1 \mathrm{~V}$. Two patients were noted to have higher thresholds $(2.88 \mathrm{mV}$ and $3.25 \mathrm{mV}$ ). (Table 2).

Conclusion In our patient cohort, use of Micra ${ }^{\mathrm{TM}}$ transcatheter pacemaker insertion was a safe alternative to conventional pacemaker insertion. The minimally invasive implantation was associated with a low acute complication rate and acceptable pacing parameters at follow-up. Longer term follow-up is ongoing.

\section{SAFETY AND EFFICACY OF 'SAME DAY' ELECTIVE CORONARY ANGIOGRAPHY AND SYNCHRONISED CARDIOVERSION IN PATIENTS WITH ATRIAL FIBRILLATION AND UNINTERRUPTED NOACS}

S Arockiam, W Mahmood, T Hennessy, T Kiernan, S Arnous. University Hospital Limerick, Limerick, Ireland

\subsection{6/heartjnl-2020-ICS.44}

Background Synchronised cardioversion is an effective means of restoring sinus rhythm in patients suffering from atrial fibrillation either in acute settings or electively after being commenced on appropriate anticoagulation. Coronary artery disease frequently coexists with atrial fibrillation. Patients with atrial fibrillation frequently undergo cardioversion and/or coronary angiography electively, possibly leading to multiple patient admissions. The safety and efficacy of having elective diagnostic coronary angiography along with cardioversion during the indexed admission with uninterrupted NOACs is not well established.

Methods A single centre retrospective analysis was performed on patients who underwent elective diagnostic coronary angiography along with cardioversion on the same day from January 2018 to December 2019. Patients had a 12 lead ECG done on the day of admission to confirm the presence of atrial fibrillation. Patients were commenced on NOACs for a minimum of four weeks prior to the day of cardioversion. Patients were specifically listed as first case in our elective cardiac catheterization laboratory. Coronary angiography was performed via transradial approach using a $5 \mathrm{Fr}$ sheath. Intraarterial cocktail of Verapamil $(2.5 \mathrm{mg})$ without unfractionated heparin was administered in each case. Hemostasis at the transradial access site was achieved by applying TR band and long protocol $(2.5 \mathrm{mls}$ slowly released every $5 \mathrm{mins}$ after four hrs of TR band application, provided there is no bleeding).

Cardioversion was performed under conscious sedation with intravenous diazepam after coronary angiography was performed in the cardiac catheterization laboratory.

Results A total of 26 patients were scheduled for elective coronary angiography and cardioversion on the same day. 2 patients were excluded; patient 1 after omission of repeated doses of NOAC and patient 2 after presence of normal sinus rhythm on admission. A total of 24 patients were eligible for the study. Baseline demographics are provided in table 1 . Normal sinus rhythm was restored in $75 \%$ of patients $(18 / 24)$. None of the patients had periprocedural complications during or after coronary angiography or cardioversion.

\begin{tabular}{|c|c|}
\hline \multicolumn{2}{|l|}{ Characteristics } \\
\hline Age (Median) & 66.5 \\
\hline Male (\% of patients) & $72 \%$ \\
\hline Female (\% of patients) & $28 \%$ \\
\hline Hypertension & $62.5 \%$ \\
\hline Diabetes & $16.6 \%$ \\
\hline Dyslipidaemia & $62.5 \%$ \\
\hline Smoking history & $62.5 \%$ \\
\hline Previous history of ischemic heart disease & $8.3 \%$ \\
\hline Average $\mathrm{CHA}_{2} \mathrm{DS}_{2}$ VASc score & 2.15 \\
\hline
\end{tabular}

Only one patient was re-admitted with symptomatic atrial fibrillation within 30 days requiring repeated cardioversion to establish normal sinus rhythm. 37.5\% of patients had concomitant moderate to severe coronary artery disease.

All patients were discharged on the same day from the day ward once the TR band was removed following the long protocol for TR band deflation. None of the patients had any immediate radial artery complication including access site bleeding or radial artery haematoma requiring intervention or represented within 24 to 48 hours with any radial artery access site complications.

Conclusion In patients suffering from atrial fibrillation who have high suspicion of underlying coronary artery disease it is safe to perform coronary angiography and cardioversion on the same day with uninterrupted NOACs.

\section{A SIMPLE QUESTIONNAIRE-BASED TRIAGE TOOL TO IDENTIFY PATIENTS POTENTIALLY ELIGIBLE FOR REFERRAL TO AN ADVANCED HEART FAILURE CENTRE}

${ }^{1} \mathrm{~L}$ Murphy, ${ }^{1} \mathrm{G}$ Giblin, ${ }^{2} \mathrm{~N}$ Caples, ${ }^{3} \mathrm{R}$ Black, ${ }^{1} \mathrm{~J} \mathrm{O}$ 'Neill, ${ }^{4} \mathrm{C}$ Halley, ${ }^{1} \mathrm{~N}$ Mahon, ${ }^{5} \mathrm{~K}$ McDonald, ${ }^{1}$ E Joyce. ${ }^{1}$ Mater Misericordiae University Hospital, Dublin, Ireland; ${ }^{2}$ Waterford University Hospital, Waterford, Ireland; ${ }^{3}$ Heart Failure National Clinical Programme, HSE, Ireland; ${ }^{4}$ St. Vincent's University Hospital, Dublin, Ireland; ${ }^{5}$ STOP-HF Unit, St. Vincent's University Hospital, Dublin, Ireland

10.1136/heartjnl-2020-ICS.45 
Introduction Accurate prevalence data for advanced heart failure $(\mathrm{HF})$, reported to range between $2-10 \%$ of the chronic HF population, are lacking. Determining the proportion of patients potentially suitable for referral to a specialist advanced HF center is crucial for accurate service planning and resource provision, particularly in the evolving era of durable mechanical circulatory support.

Purpose To identify the population of patients potentially eligible for referral for assessment for advanced surgical therapies to the National Advanced HF and Cardiac Transplant center, using a quick one-page prospective survey.

Methods A survey comprising 13 potential markers of advanced HF was developed, modified from the 2018 position statement of the Heart Failure Association of the European Society of Cardiology. This was distributed to $26 \mathrm{HF}$ clinic centers nationally. Each center was asked to complete the survey on consecutive patients over a 3 -month period who fulfilled the following three criteria: 1) age $<65$ years; 2) ejection fraction (EF) $<40 \%$ and 3 ) HF of $>3$ months duration.

Results In all, 21 of $26 \mathrm{HF}$ clinic centers participated in the survey. Across the period of inclusion, 4950 all-comer HF patients were seen in 21 centers. Of these, 375 fulfilled the inclusion criteria, comprising $279(74.4 \%)$ males with a median age of 57 years. In total, 246 (66\%) of the completed surveys had one or more potential markers for advanced HF, representing just under $5 \%$ of the total all-comer HF population seen across the same time period. Of these, 67 patients $(27 \%)$ had at least two, $48(20 \%)$ had three and $40(16 \%)$ had $\geq 4$ potential markers. The most frequently noted markers were $\geq 1$ hospitalization or unscheduled clinic review (56\%), intolerance to renin-angiotensin-aldosterone system inhibitors due to hypotension or renal dysfunction (29\%) and intolerance to beta-blockers due to hypotension (27\%). Almost one-quarter of patients reported NYHA Class III or IV symptoms (figure 1).

Conclusions In this index prospective National survey, approximately $5 \%$ of an all-comer routine $\mathrm{HF}$ clinic population and two-thirds of a pre-selected HF with reduced EF $<65$ years cohort, were found to have at least one clinical or biochemical marker suggesting advanced or impending advanced HF. Notably, almost one-quarter of patients in this chronic outpatient 'snapshot' population have advanced HF symptoms. This simple one-page triage tool may be useful to identify a population potentially eligible for referral to an advanced $\mathrm{HF}$ center for assessment for advanced surgical therapies, thereby aiding resource utilization and service planning.

\section{THE PROFILE AND OUTCOMES OF PATIENTS REFERRED TO THE NATIONAL ADVANCED HEART FAILURE OUTPATIENT CLINIC}

L Murphy, G Giblin, C Tracey, C Howley, E Kavanagh, J McGuinness, N Mahon, E Joyce. Mater Misericordiae University Hospital, Dublin, Ireland

\subsection{6/heartjnl-2020-ICS.46}

Introduction The burden of advanced heart failure (HF) is an understudied aspect of Ireland's chronic HF population. Given an expanding menu of advanced HF therapies, identifying suitable candidates for timely assessment and intervention with progression to heart transplantation or mechanical circulatory support (MCS) if appropriate, is imperative. However, given the intensity of resource and service utilization involved, contemporaneous epidemiological and descriptive data is essential for adequate resource planning and provision.

Purpose To identify demographics and outcomes of patients referred to the National Advanced HF and Cardiac Transplant centre, including identifiable markers of advanced HF according to the Heart Failure Association of the European Society of Cardiology (ESC) 2018 position statement.

Methods Consecutive patients referred to the national advanced HF clinic over a 9-month period from May 2019 to February 2020 were prospectively included in this registry. Baseline demographics, laboratory, electrocardiogram, echocardiogram parameters were recorded. Markers for advanced HF according to the ESC 2018 'I NEED HELP' criteria (table 1) were documented for each patient. Outcomes including need for admission from outpatient clinic, requirement for inotropes or subsequent MCS and/or heart transplantation and mortality were recorded.

Results A total of 32 patients were enrolled (28\% female, mean age: $49 \pm 12$ years, $50 \%$ NYHA III or IV) over the 9month period. The vast majority $(81 \%, n=25)$ had at least one HF hospitalization in the preceding year, with $25 \%$ $(n=8)$ having $\geq 2$. Frequency of markers of advanced HF,

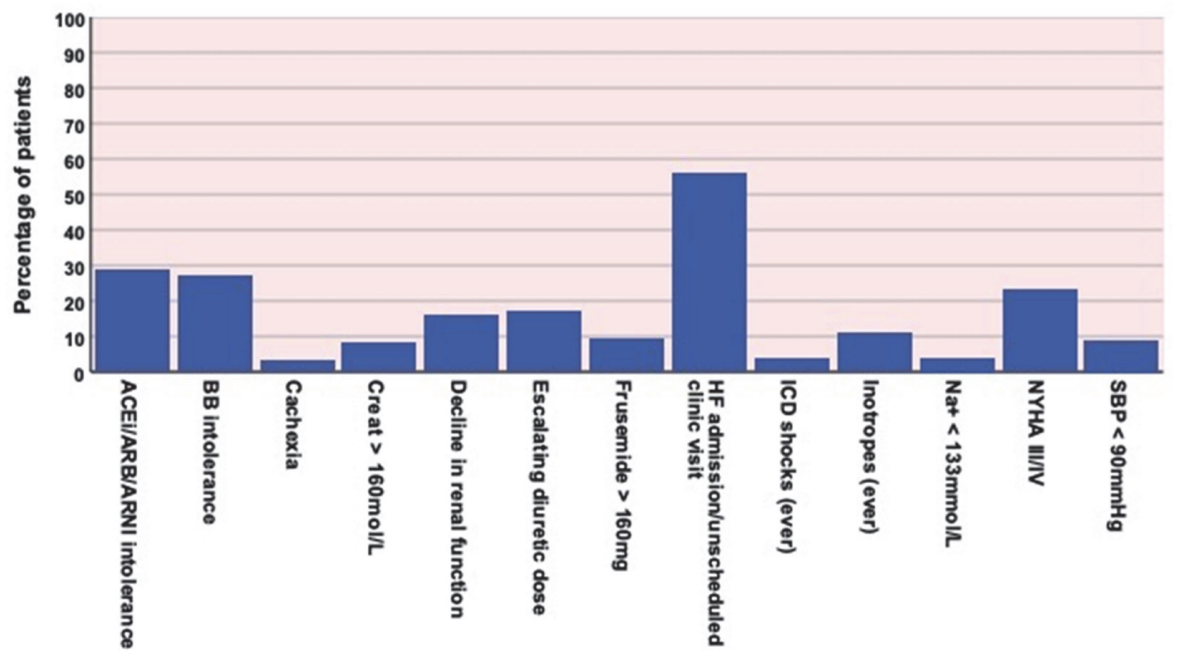

Abstract 45 Figure 1 NYHA Class III or IV symptoms 